\title{
Automatic coronary artery segmentation based on multi-domains remapping and quantile regression in angiographies
}

\author{
Zhixun Li $\mathrm{Li}^{\mathrm{a}, \mathrm{b}}$, Yingtao Zhang ${ }^{\mathrm{a}}$, Huiling Gong ${ }^{\mathrm{b}}$, Weimin $\mathrm{Li}^{\mathrm{c}}$, Xianglong Tang ${ }^{\mathrm{a}}$ \\ ${ }^{a}$ School of Computer Science and Technology, Harbin Institute of Technology, China \\ ${ }^{b}$ School of Information Engineering, Nanchang University, China \\ ${ }^{c}$ The First Affiliated Hospital, Harbin Medical University, China
}

\begin{abstract}
Coronary artery disease has become the most dangerous diseases to human life. And coronary artery segmentation is the basis of computer aided diagnosis and analysis. Existing segmentation methods are difficult to handle the complex vascular texture due to the projective nature in conventional coronary angiography. Due to large amount of data and complex vascular shapes, any manual annotation has become increasingly unrealistic. A fully automatic segmentation method is necessary in clinic practice. In this work, we study a method based on reliable boundaries via multi-domains remapping and robust discrepancy correction via distance balance and quantile regression for automatic coronary artery segmentation of angiography images. The proposed method can not only segment overlapping vascular structures robustly, but also achieve good performance in low contrast regions. The effectiveness of our approach is demonstrated on a variety of coronary blood vessels compared with the existing methods. The overall segmentation performances si, fnvf, fvpf and tpvf were $95.135 \%, 3.733 \%, 6.113 \%, 96.268 \%$, respectively.
\end{abstract}

Keywords: Reliable boundaries, Multi-domains remapping, Quantile regression, Coronary artery segmentation, Angiography image

\section{Introduction}

The heart is one of the most important organs in human body. And the coronary artery, which can provide oxygen for heart, is the most important 
blood vessels. Nevertheless, coronary artery disease (CAD) is a leading cause of death worldwide[1]. Although many new technologies have come out such as the CTA and MRI, conventional coronary angiography (CCA) is still the most common way of screening for CAD due to its process speed and accuracy. In clinic practice, the segmentation of accurate vascular structures holds great significance in describing the potential implications for the anatomy and diseases, it is an essential step in stenosis detection, vascular shear stress estimation, and image registration [2][3][4]. It provides the coronary arteries, branches, twisted and other anatomical information, as well as other pathological information such as calcified plaque and stenosis. Moreover, a robust coronary artery segmentation is the basis of many post-processing visualization tools, for example, curved multi-planar reconstruction (curved MPR). Due to image noise, artifacts, non-uniform illumination in angiographies, the performance varies easily with the image quality. Furthermore, vascular structures also contain a variety of complex types: overlapping, crossing or bifurcation. Therefore, a robust and accurate segmentation is a grand challenge.

Methods of vessel segmentation have been proposed in numerous publications[5], which can be divided into three categories mainly: Image intensity-based, statistical model-based and others. Gradient-based modeling refers edge pixels determined by the large gradient through the first or second order derivative calculation. Classical edge detection operators are: Roberts edge operator, Sobel edge operator, Prewitt edge operator, Laplace edge operator, GaussLaplace and Canny edge operator. Another popular vascular segmentation method is based on Hessian matrix, which is like a filter to responde the local linear features, such as the Lorenz's vesselness[6], the Sato vesselness[7], the Frangi's vesselness[8] and its improved method[9]. However, these methods cannot perform well on the small blood vessels. They are sensitive to local deformation and noise because local linear signal may be responded easily. And another trainable filter is called the combination of Shifted Filter Responses(COSFIRE)[10], which is weak in the case of complex texture. Active contour model [11][12] has also been used for blood vessels segmentation. They are based on an energy minimization model under the internal and external forces with the object boundary contour evolution. It can provide a framework to integrate different features and models. Graph Cut (GC) technique can rely on a contextual analysis model between foreground and background pixels[13][14][15], both the regional and the boundary properties can be used. However, this method may be affected by "shrinking bias" 
problem which may deviate to small part of the isotropic region, since its energy value is proportional to the length of the border and results[16].

Statistical model-based segmentation methods are another popular class. All of them require a model based on the statistics of medical features. One of these statistical models is active appearance model (AAM)[17], establishing the spatial relationship between respective appearance pixels in all examples. Therefore it can be employed for global shape constraints and subsequent image analysis tasks, and can combine with active shape model (ASM), which is the relationship between respective shape landmark points [18] for constructing a hybrid model[19][20]. Although their robustness to artifacts and noise have been proven, some drawbacks(over-fitting and limited generalization) are inevitable. Due to non-uniform illumination of contrast agent, the different shapes of vessels, presence of calcification or plaque in blood vessels, diverse CCA image forms may be produced. Moreover, it is more difficult for acquisition of all kinds of training examples, even it is impossible. And manual annotation of foreground and background as a training set cannot be very precise, because of the sample complexity, labeling experience, etc.

There are other segmentation methods tracking[21][22][23], atlas-guided[24] and phase congruency[25]. Tracking is the easiest way to obtain specific region criteria, whose boundary pixels are gradually introduced through the tracking process. Classic tracking technique is suitable for detecting high contrast blood vessels, however for the unclear vessel, segmentation will be easily spreaded to surrounding tissue due to some pathological changes, lowcontrast or noise. In order to deal with such problem, atlas-guided registration is introduced to register a labeled atlas, which describes an overall structure in a given type of image. Then the image is segmented by obtaining a transformation parameters to the atlas. Obviously, a general atlas is not natural to be found. Phase congruency method is based on the human visual system, and it works by keeping sensitive to high phase consistency on visual boundaries. Therefore it can obtain more accurate border locations and richer useful underlying boundary features due to its invariability to the image brightness and contrast. Although phase congruency can overcome some drawbacks, the enhancement of artifacts will result in additional artifacts interference.

Overall, the coronary artery segmentation is a very challenging issue because of their various shapes, appearances, different lengths, and a variety of branch location. More importantly, the projective imaging causes very complex texture and serious overlapping interference, especially for the low 
contrast regions. Therefore, the methods based on intensity and frequency domains are difficult to detect their edges accurately in this case. For the statistical learning methods, because of the inevitable human intervention, they may lead to false segmentations. A serious obstacle is the robustness of parameter estimation methods which are correlated with heteroscedasticity, because homoscedasticity is a key assumption to guaranty good statistical properties for these methods. If this assumption is not met, their results will be infinitely affected.

In our work, the main components of this approach consist of the boundary combination of intensity and frequency, profile construction, distance discrepancy correction and quantile regression. Firstly, the remapping of two domains is used to extract the reliable edges. In general, there are two kinds of boundary extraction methods, the strength based and frequency based. Frequency based method can avoid some artifacts in the strength based methods through phase information. A distance function based on minimum Baddeley Delta Metric (BDM) [26] is constructed to obtain common salient edges in two domains. Next, a profile along the centerlines sampling points can handle the edges pixels independently so that a global threshold segmentation can be avoided. Finally, distance discrepancy correction and quantile regression are employed to obtaining accurate boundaries as closely as possible. Quantile regression is the key factor compared with the traditional regression methods based on the mean value such as OLS, it has the following advanced characteristics[27]. (1) The quantile regression has strong adaptability, because it needs not to require the model residual distribution. (2) Interdependent relation on all the quantiles can be analyzed, hence outliers can be isolated robustly. (3) With the aid of multi quantiles, the quantile regression can achieve more details in relationship between the dependent and independent variables. These characteristics determine that the piece-wise regression can be more robust than ordinary regression. The results show the effectiveness in processing various coronary blood vessels compared with existing methods. The flowchart of the proposed method is shown in Fig.1.

The remaining part of the paper is organized as follows: Section 2 describes the proposed coronary artery segmentation method. Experimental results are demonstrated in Section 3. Finally, Section 4 is for discussions, conclusions and future prospects. 


\section{Proposed method}

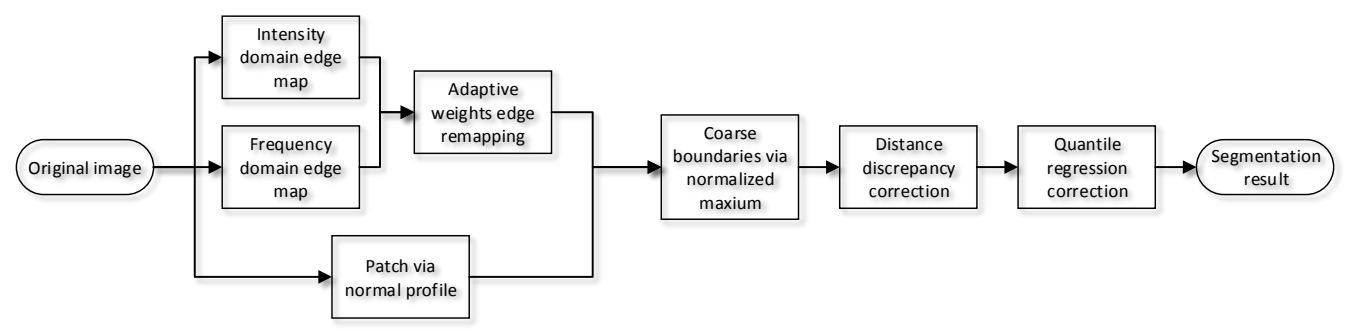

Figure 1: The flowchart of the proposed coronary arteries segmentation algorithm

The proposed method initiates from a vessel centerline, which can be obtained from an existing fully-automatic, semi-automatic or manual technology. In this work, a fully automatic procedure is established based on previous findings on centerline extraction[28].

The centerline can be extracted through a robust connectivity and principle curves, a brief description is given as follows. It mainly consists of three steps mainly: (1) a vesselness compensation and binary thresholding on Frangi's vascular filter can ensure connectivity and eliminate artifacts as much as possible. Vessels with branches are thus separated into multiple independent segments, which are without any branch. (2) A connectedness cost function is defined according to correlation of vessel segments to identify the same segments belong to one vessel. There are three terms: Directional continuity. Directional continuity on adjacent segment's endpoints is a key factor to determine whether the segments belong to one blood vessel or not. It can be advisable to judge the matching degree between broken parts in one vessel according to their directions. However, due to some large gaps, it is difficult to match exactly directions of the two parts. End points' distance are introduced to compensate direction's drawback. Distance between end points. We introduce an energy distribution filed from segment-end with a radius and angle's range. More specifically, a coverage area is constructed by a radius and a sector based on Gabor filter. And then a decay function is defined as the energy's strength varies with the distance from end points. Difference of widths between two vessels on endpoints. In general, the vessel width changes slowly in a short segment, so the difference of width between two vessel segments represents the third factor for determining if they belong to one vessel. (3) In order to obtain robust centerline and overcome 
the influence of break-offs and holes, a method based on principal curves is employed to extract centerline.

On the basis of the centerline aforementioned, the reliable boundaries can be extracted via remapping of multi-domains, and border pixels can be filtered by distance discrepancy correction and quantile regression. In order to promote computational efficiency, coarse region of vessel (ROV) is delineated beforehand by profiles of appearance model based on normal as a patch.

\subsection{Vessel Location and Coarse Region of Vessel}

Obviously, the reduction of calculating area can be helpful to improve the efficiency and eliminate the interferers of mostly artifacts, because the vascular region only occupies a small fraction of the entire image. Next, patch can be delineated by the normal vector with an appropriate length along the centerline as the coarse region of vessel. Specially, the pixels on the initial centerline are sampled with equal length $\Delta d$ according to the actual demand, and a set of $L$ points is generated. Normal vector to each landmark $\left(x_{n}, y_{n}\right)$ can be calculated from the adjacent points $\left(x_{n-1}, y_{n-1}\right)$ to $\left(x_{n+1}, y_{n+1}\right)$.

On both sides of the centerline, $k$ pixels are sampled by using a fixed distance $k$ along the normal direction for each landmark, it can give a patch of length $2 k+1$. Finally all pixels in respective patch can be used to get a graylevel appearance model profile including edge salient map and correspondent coordinates around the vessel.

In order to ensure the accuracy, we separate the centerline into disconnected segments. It should be noticed that intersectional points of the centerline has no obvious relationship with their predecessors or successors. Hence, the normal vector at the intersection cannot be successfully calculated. Therefore the front and rear points are ignored.

\subsection{Reliable Boundary Extraction}

A good training process requires the reliable boundaries. Currently, most edge extraction algorithms are based on the gradient calculation of pixel intensities. Gray-scale gradient-based approach is widely employed in edge detection methods. However, due to the non-uniform illumination of contrast agent and projective imaging, the overlapped texture is very complex in CCA image, and the traditional intensity methods cannot extract the edges very well. 
In order to achieve the robustness in boundary detection, the intensity and frequency domains are carried out simultaneously in the proposed method. The frequency domain based boundary detection is independent to the intensity, hence it can take the advantages and bypass the disadvantages with the methods based on intensity. We address the reliable boundary extraction as a minimum quantitative measure of the difference between intensity and frequency edge saliency. They are as follows:

Intensity domain. Classical Sobel operator is employed as the intensity method. The brightness gradient can be calculated in two dimensions, and the gradient map is generated for all pixels from the original image.

Frequency domain. Frequency domain detection is based on phase congruency, which is invariant to the brightness and contrast, therefore it is an important indicator of edge detection[29]. Phase congruency model assumes that the salient points are at the positions where phases are highly consistent on all Fourier components[30]. It can be described as follows:

$$
f(x)=\sum_{n} A_{n} \cos (n \omega x+\phi),
$$

where $f(x)$ is the Fourier Series, $A_{n}$ is the amplitudes of $n$ Fourier components, $\phi_{n}(x)=n w x+\phi$ represents the local phase at $x$. The phase congruency can be defined as:

$$
P C(x)=\max _{\theta \in[0,2 \pi]} \frac{\sum_{n} A_{n} \cos \left(\phi_{n}(x)-\theta\right)-t}{\sum_{n} A_{n}+\epsilon},
$$

In order to make $P C(X)$ obtain the maximum value at scale $n$ and orientation $\theta, \phi$ is set to a weighted average whose $A_{n}$ are the weight: $\bar{\phi}(x)=\sum_{n} A_{n} \phi_{n}$. Since $\cos (x)$ approximately equals $1-x^{2} / 2$ in Fourier expansion, the positions with the highly consistent phase are approximately equal to the points with the small local phase variance. And $t$ is a noise threshold, $\epsilon$ is a small real number used to avoid division by zero.

The Log Gabor is able to deal with the image having wide brightness range. And it has other advantages covering a large range of frequencies, reducing the amount of calculation, and being consistent with human visual system metrics. Therefore, Log Gabor is usually used as a filter group for consistency analysis and calculation: 


$$
\begin{aligned}
& g(\omega)=\exp \left\{\frac{-\left(\log \left(\omega / \omega_{0}\right)\right)^{2}}{2\left(\log \left(\beta / \omega_{0}\right)\right)^{2}}\right\}, \\
& \omega_{0}=1 / \operatorname{minWaveLength} * \text { mult }^{(s-1)},
\end{aligned}
$$

where $s$ is the scale, minWaveLength is the length of the smallest scale filter, and mult represents the scaling factor between successive filters. $\omega_{0}$ is the center frequency of the filter, and $\beta$ controls the frequency of the filter.

Quantitative measure remapping. Each of these two domains has its own advantage and disadvantage, but we need a tradeoff between them. It can be regarded as obtaining respective weights through minimum distance optimization. Baddeley Delta Metric (BDM) is introduced as the distance for the two edge maps. Its distance is inspired from Hausdorff distance[26]. It holds some desirable properties and generates a myopic topology. It is given by the following formula:

$$
w^{*}=\underset{w}{\arg \min }\left[\frac{1}{|P|} \sum_{p \in P}\left|w d\left(p, E_{\text {intensity }}\right)-(1-w)\left(d\left(p, E_{\text {frequency }}\right)\right)\right|^{k}\right]^{1 / k}
$$

where $w$ is the weight for trade-off between two domains and $p \in \mathbb{R}$, $1<p<\infty$ and it allows to adjust the value of the point-to-point distances. The parameter $k$ influences the evolution of $\Delta_{w}^{k}$ with respect to the image degradation.

Moreover, in order to avoid a global threshold, we regard a boundary point as the maximum in respective profile of each landmark. As for the profile of gray appearance model, Cootes and Taylor recommended that normalization is used with first order derivatives, which is the sum of their value of all elements equals one[17].

\subsection{Distance discrepancy correction}

Although the "reliable" boundaries have been extracted, they are just computed edges without any meaning to medicine. The artifacts' boundaries are also introduced, however they are not real vessels at all. In order to distinguish the vascular and artifacts' boundaries, we need to filter out the points which do not belong to vessels. Especially on some vascular branch or missing regions, satisfactory results are harder to be obtained. We still want to guarantee the results as close as doctor might expect, this issue is 
regarded as the distance discrepancy correction. The distance is considered as the length between the boundary point and its corresponding point on the centerline in the respective profile. In addition, this step can improve the accuracy and reduce the computational complexity further for the following intensity regression.

A continuity measure is used to judge whether there exists a discrepancy via the coordinates of one's adjacent precedent and successive points, which are set as $i_{\text {pre }}$ and $i_{\text {suc }}$ and a good judgement can be performed for the artifacts with some consecutive points. Specially, the maximum between one point's precedent and successive $n$ points is regarded as the distance discrepancy metric $D_{d i s}$, as following:

$$
D_{\text {dis }}=\max \left(\frac{1}{n} \sum_{i_{\text {pre }}=1}^{n}\left(D_{c}-D_{i_{\text {pre }}}\right)^{2}, \frac{1}{n} \sum_{i_{\text {suc }}=1}^{n}\left(D_{c}-D_{i_{\text {suc }}}\right)^{2}\right),
$$

where the current point is $D_{c}, i_{\text {pre }}$ and $i_{\text {suc }}$ represent its precedent and successive points, respectively.

In order to discriminate normal and abnormal points, a threshold is introduced. The threshold does not require a very precise value, but it can should be set as large as possible, because we would rather misjudge some right points than let wrong points get off. In the correction step, we only refer to these normal points, which are at appropriate range of distance by thresholding. And the correction value $D_{\text {corr }}$ is set to the average of them:

$$
D_{\text {corr }}=\frac{1}{n} \sum_{P_{N}} D_{N}
$$

where $P_{N}$ and $D_{N}$ represent the normal points and their distances, respectively.

Finally their coordinates are obtained in their profiles.

\subsection{Robust Boundary Refinement with Quantile Regression}

For further refining the real vascular boundary positions, multiple linear regressions can be applied for modelling the relationship between the intensity on the central area of the vessel and the intensity on the vessel boundary. The purpose of this step is to adjust refinement the inconsistent points on the boundary through intravascular intensity. 
Although there are many regression methods such as ridge regression, multivariate linear planning, most of them are based on the conditional mean model. In the past half century, the least squares method and its derived methods are considered as the most important modern statistical tools. However, in this case, the assumption of homogeneity of variance is violated frequently. Moreover, some useful information may be ignored if we only focus on the central place of the distribution of the dependent variable. Their regressions may be inappropriate and misleading, and the robustness is difficult to keep, because the conditional mean may be interfered by the outliers in the presence of heteroscedasticity.

However, regression robustness can be greatly improved through quantile regression, which will not be disturbed by the outliers through ignoring the homoscedasticity assumption. Given any real valued random variable $X$, which can characterized by its distribution function:

$$
F(x)=\operatorname{Pr} o b(X \leq x)
$$

for any $0<\tau<1$

$$
Q^{-1}(\tau)=\inf \{x: F(x) \geq \tau\}, 0<\tau<1
$$

which is called the $\tau$ th quantile of $X . Q^{-1}(0.5)$ is the median.

As in the least squares, a sample mean is regarded to solve the problem

$$
\min _{\xi \in R} \sum_{i=1}^{n}\left(y_{i}-\xi\right)^{2}
$$

which suggests that the conditional mean of $y$ given $x$ can be expressed as $\mu(x)=x^{\prime} \beta$, and $\beta$ is estimated by solving

$$
\hat{\beta}=\arg \min _{\beta \in R}{ }^{k}\left\{\sum_{i=1}^{n}\left(y_{i}-x_{i}^{\prime} \beta\right)^{2}\right\} .
$$

Similarly, consider the $\tau$ th sample quantile, $\hat{\alpha}(\tau)$ solves

$$
\min _{\xi \in R} \sum_{i=1}^{n} \rho_{\tau}\left(y_{i}-\alpha\right),
$$


and the $\tau$ th conditional quantile function is expressed as $Q_{y}(\tau \mid x)=x^{\prime} \beta(\tau)$ and $\hat{\beta}(\tau)$ is solved by

$$
\left.\hat{\beta}=\arg \min _{\beta \in R} k \sum_{i=1}^{n} \rho_{\tau}\left(y_{i}-x_{i} \beta_{\tau}\right)\right\} .
$$

A linear function can be minimized on a polyhedral constraint set, and the important properties of the solutions $\hat{\beta}(\tau)$, regression quantiles, are followed from the well-known properties of solutions of linear programs. The quantile regression problem can be reformatted as a linear programming

$$
\min _{\xi \in R}\left\{\sum_{i: y_{i} \geq \xi} \tau\left|y_{i}-\xi\right|+\sum_{i: y_{i} \leq \xi}(1-\tau)\left|y_{i}-\xi\right|\right\}
$$

The regression robustness has been enhanced by minimizing the distance equation (13). Specially, if we modify the dependent variable which lies above (or below) the quantile regression line corresponding to a certain data, the regression line will be unchanged, i.e., this data point is still above (or below) the regression line. In other words, the regression line will remain unchanged if we only modify the values of dependent variable without changing the sign of their residuals. Therefore, the influence of the outliers is very limited. Furthermore, the quantile regression's distributional hypothesis is very robust, because its estimation is more dependent on the distribution nearby a specific quantile instead of distribution points far from this quantile, so quantile regression line is not affected by the overall distribution.

In addition, quantile regression can fit a bunch of curves. And when the independent variables have different distributions in different parts of the dependent variable, quantile regression can provide more robust and comprehensive information of conditional distribution.

\section{Experiments and results}

In order to validate the performance of the proposed method, we test it using clinical angiographies.

\subsection{Materials}

We build a dataset, which consists of 60 coronary angiography images from 34 patients, acquired with GE INNOVA 2000 and Philips Allura Xper 
FD10 detector from the First Affiliated Hospital of Harbin Medical University. The boundaries of coronary arteries have been blindly annotated by three experts, and there is no information exchange between them. The experts segmented all vessels manually and extracted the centerlines assisted by semiautomatic segmentation algorithm (QCA-CMS Version 6.0, MEVIS, Bremen, Germany). The proposed method is implemented with Matlab 2013a. Experiments are performed on a standard computer with $3.2 \mathrm{GHz} \mathrm{CPU}$ and 8 GB of RAM.

\subsection{Evaluation Measures}

we consider the result as a pixel-based classification, a pixel can be classified as blood vessels or non-vessels. The following criteria from [31] are used to evaluate the performance of the proposed algorithm. Let $L_{\text {org }}$ be the ROI of the ground truth boundary and $L_{I}$ is the ROI of the proposed segmentation boundary. We define the set of false negative pixels $f n_{I}=L_{\text {org }}-L_{I}$, the set of false positive pixels as $f p_{I}=L_{I}-L_{\text {org }}$ and the set of true positive pixels as $t p_{I}=L_{\text {org }} \bigcap L_{I}$. By using these definitions, we derive the following four measures : false negative volume fraction $(f n v f)$, false positive volume fraction $(f p v f)$, true positive volume fraction $(t p v f)$, and similarity index (si) :

$$
\begin{gathered}
f n v f=\frac{f n_{I}}{L_{\text {org }}} \\
f p v f=\frac{f p_{I}}{L_{\text {org }}} \\
t p v f=\frac{t p_{I}}{L_{\text {org }}} \\
s i=1-\frac{f n_{I} \cup f p_{I}}{L_{\text {org }} \cup L_{I}}
\end{gathered}
$$

In our experiments, they are calculated over all the test images.

\subsection{Parameter Setting}

Region of interest. Larger profile lengths may cause more overlapping among profiles and may generate more interferences in the subsequent process. However, if it is too small, blood vessels cannot be contained in the profile. In consideration of the maximal vessel radius is 10 pixels in our dataset, we 
set the profiles radius to 12 pixels with an error of approximately 2 pixels. And according to the image resolution and experimental analysis, pixels being sampled every 3 pixels can balance both of good centerline shape and computing performance.

Phase congruency. The scale $s$ is set to the ranges from 1 to 4 . Scale selection is based on the maximum and minimum blood vessels diameters. The scale range $s$ is thus set to from 1 to 4 referred to vessels radii varied from 1 to 10 pixels in our database. For the calculation of $\omega_{0}$, minWaveLength, mult are set to 3 and 2.1 , respectively. $\beta / \omega_{0}$ is set to 1.2 . The number of filter orientations is 6 . The $t$ is calculated by employing the median of the smallest scale filter responses to estimate noise level. The parameters are manually selected through the optimization for providing the best visual effect. Due to the similarity in angiography images, fine-tuned parameters will slightly change their results. Its details are described in [28].

Distance correction. Obviously, the more reference points will correspond to the longer contour. The farther away reference point is, the lower correlation is with the correct point, and the nearer reference point is, the corrected value will be affected more by noise. In order to make a balance, we set 3 points. And in views of vascular change of radii and the influence of the inaccurate centerline, the distance threshold is set as 2.5. This value is just the constraints for the subsequent quantile regression, therefore the precision of this value will affect the final result slightly. And it is validated in the experiments.

Quantile regression. The quantile $\tau$ is a number strictly between 0 and 1 , and they are listed in $\{0.05,0.1,0.25,0.5,0.75,0.9,0.95\}$. The fitting method is based on Frisch-Newton interior point method and it may be used to compute the full quantile regression process efficiently. Although more quantiles can achieve more detailed information, computational efficiency has to be taken into account. In fact, more quantiles will greatly increase the computing time. Because there is no extreme scattered distribution between strength within vessel and strength on the boundary, the distinction between adjacent quantile is not large. Therefore we only consider seven quantiles, their intervals are more closely at the both ends (0.05 quantile) and are relatively dispersed in the center (0.25 quantile).

\subsection{Results}

The illustrative segmentation results in each step are shown as follows. 


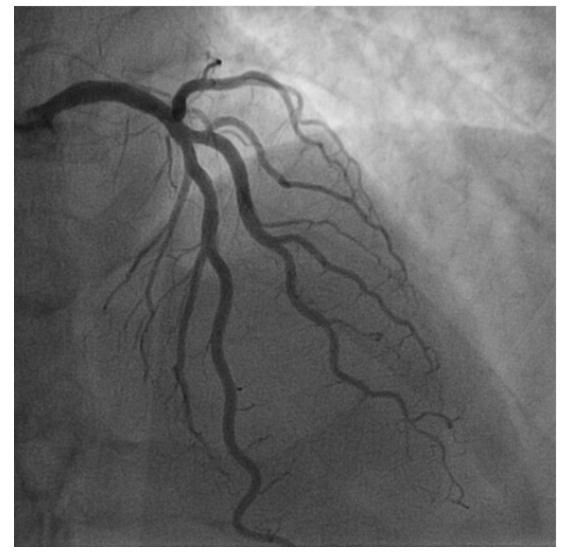

(a)

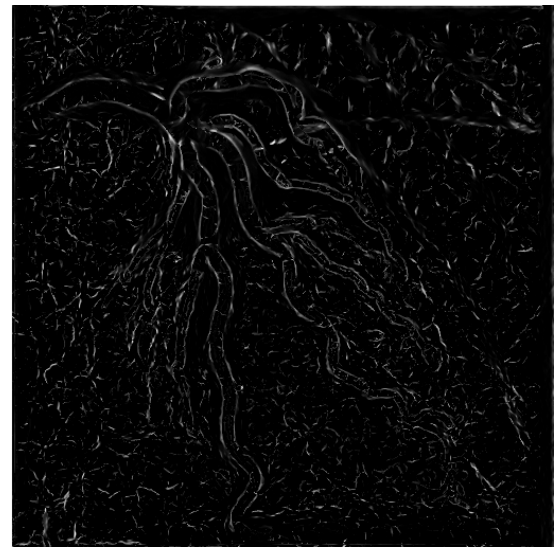

(c)

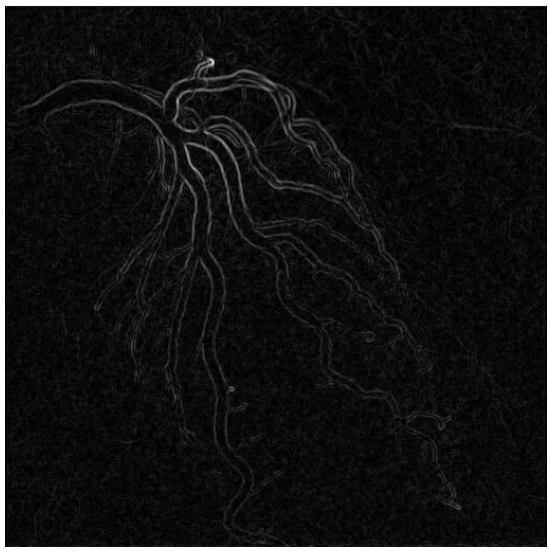

(b)

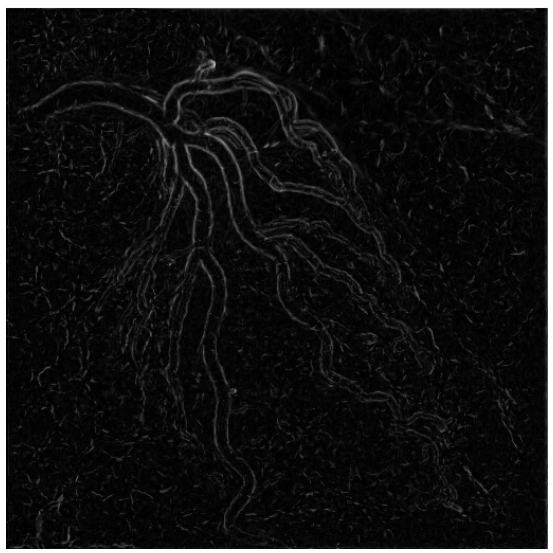

(d)

Figure 2: Remap of two domains: (a) Original, (b) Sobel boundary operator, (c) Phase congruency boundary operator, (d) Combination of two operators 


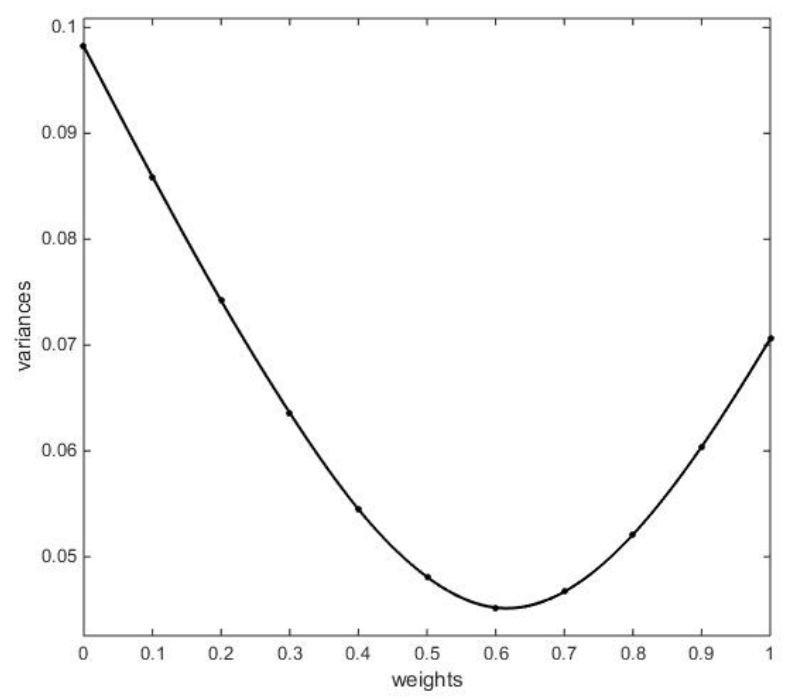

Figure 3: Variances vs weights between intensity and frequency domains

Fig.2 shows a multiple domains remapping process of a clinical image. Fig.2(b) is a gray gradient map in the intensity domain. The boundaries can be well delineated for the clear regions, but due to its intensity-dependent nature, it may lead confusion between vessel and artifact, which have the same signal gradient. This operator may be in trouble with a general threshold on segmentation and the vessel borders may be missing. As for phase congruency in frequency domain, artifacts are markedly increased as shown in Fig.2(c) due to its strength independency on boundaries extraction. However, certain vessel boundaries, which are weak in intensity domain as shown in Fig.2(b), are significantly enhanced simultaneously. Fig.2(d) shows our weighted remapping in intensity and frequency domains. Its plot of variances vs weights is shown in Fig.3. And the best weights, which can minimize the difference of edge salient map in the two domains, can be observed in this figure. In remapping figure, its artifacts' boundary saliency can be alleviated than Fig.2(c), and the blood vessel boundaries can be more balanced than Fig.2(b).

The patch consists of all profiles along the normal direction via the calculation on two adjacent points as shown in Fig.4(a). And we can observe that 


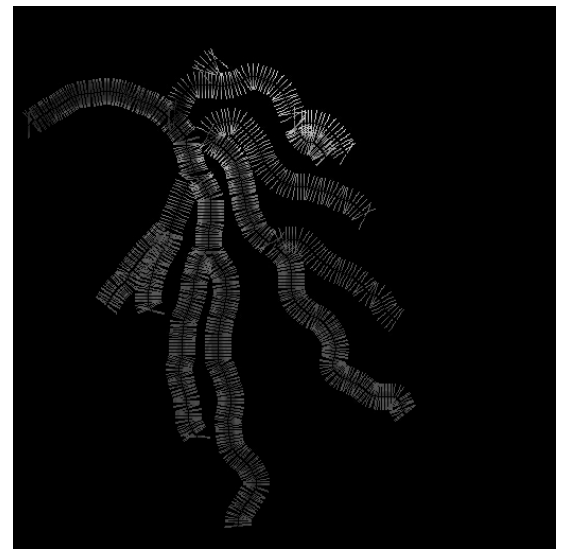

(a)

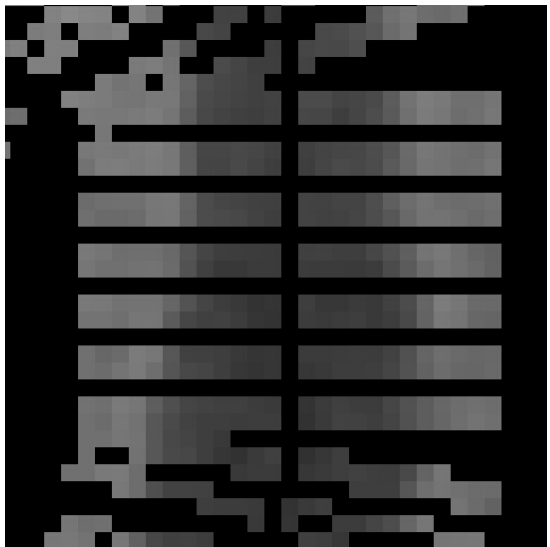

(b)

Figure 4: Patch via normal direction: (a) Normal patch, (b) Zoomed patch

the endpoints are inside the portion of the blood vessels, hence the endpoints' profiles are useless and ignored during the following process. Fig.4(b) is a zoomed figure of a normal profile in term of one segment of blood vessels. In this paper, the coarse boundary points are regarded as the normalized maximum. Obviously, calculation of each profile is more simple and effective than a global threshold setting on entire image.

Fig.5 shows the distance discrepancy distribution through a reference to adjacent points on both side of coarse vessel boundary along the centerline. As we all have observed, in the most regions, the boundaries consistent with the really borders. Due to transmission nature in the angiography image, there are some positioning errors especially on the branches, other organ texture and tiny blood vessels. The plot in Fig.5(b) shows the distance distribution along the continuous adjacent boundary points. According to the experiments, a threshold value is set to 2.5. All points beneath this value are considered that there is a good match with the boundary conditions. It is worth pointing out that this value does not require a precise setting, because the next step will conduct a precise adjustment through intensity regression, so the main purpose of this step is to improve the computational efficiency.

Fig.6(a) shows a bunch of regression lines on all levels of quantile. The plot shows a scatterplot of the intensities on boundaries vs centerlines. And quantile regression lines are gray, the median regression line is solid black, and least squares regression is based on conditional mean function as dashed red. 


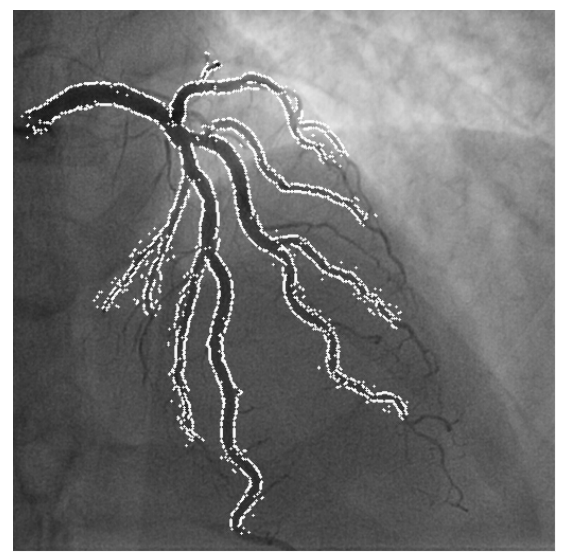

(a)

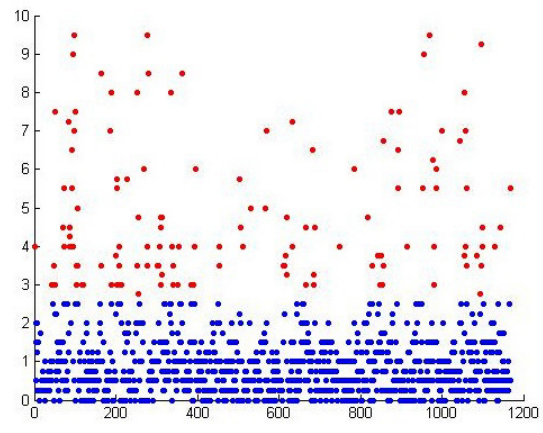

(b)

Figure 5: Distance distribution: (a) Coarse boundaries, (b) Distance deviation

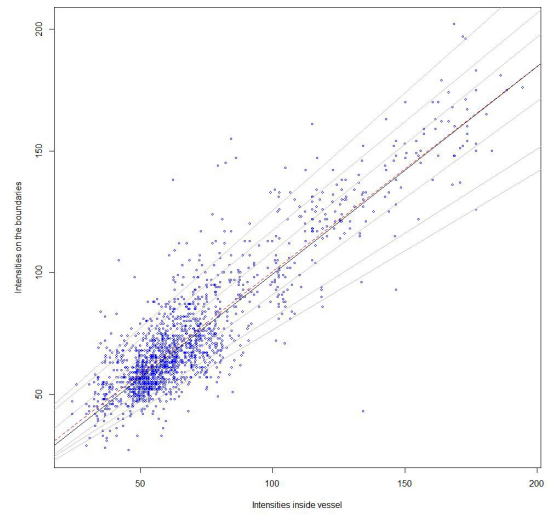

(a)

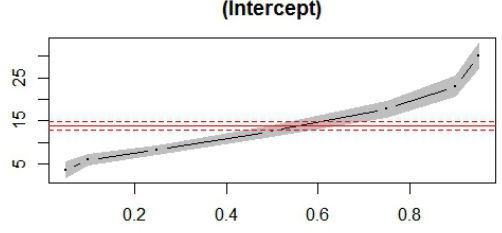

$\mathrm{x} 1$

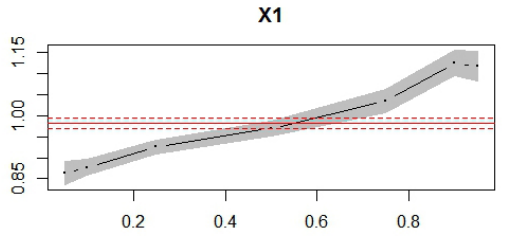

(b)

Figure 6: Quantile regression: (a) Scatterplot and Quantile Regression Fit, (b) Coefficient Plots 
Table 1: Estimated intensity vaule comparison of quantile regression and original least square in quantiles

\begin{tabular}{|l|l|l|l|l|l|l|l|}
\hline Quantiles & 0.05 & 0.1 & 0.25 & 0.5 & 0.75 & 0.9 & 0.95 \\
\hline Intensity inside vessel & 39 & 45 & 51 & 59 & 73 & 102 & 126 \\
\hline Ground truth on boundary & 42 & 49 & 53 & 63 & 84 & 114 & 138 \\
\hline Intensity on boundary(QR) & 37 & 44 & 52 & 64 & 85 & 119 & 150 \\
\hline Intensity on boundary(OLS) & 48 & 54 & 59 & 65 & 77 & 102 & 122 \\
\hline
\end{tabular}

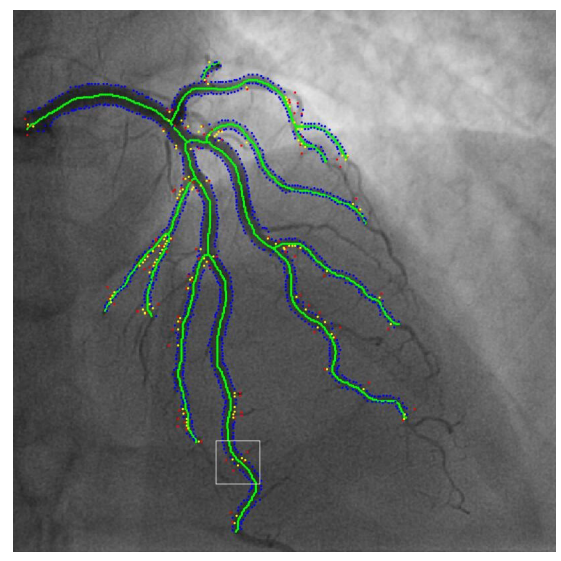

(a)

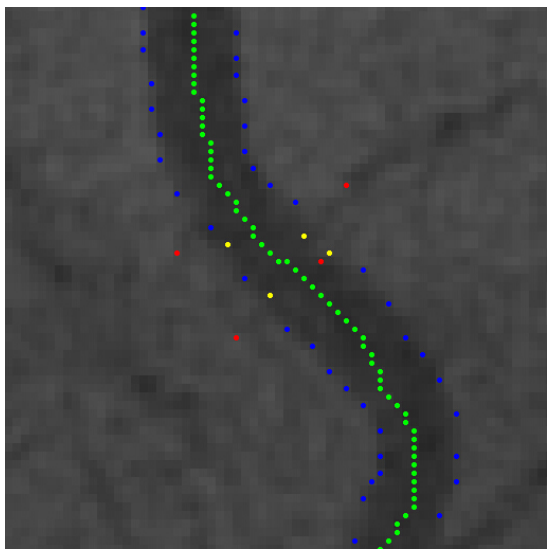

(b)

Figure 7: Corrections: (a) Corrected points, (b) Zoomed region. The discrepant points, corrected points and centerline points are in red, yellow and green, respectively.

It can be observed that all lines are distinct, and their intercepts and slopes can be seen clearly from Fig.6(b) on different $\tau$. The slope increases gradually with the higher quantile, it shows that the strength of the boundary point varies according to the intensity of the central point. The red line represents the mean's location. We can observe that in Table 1. Compared with OLS, our estimated intensity values are closer to the ground truth. And in this image, the boundary intensity variable has been centered at its mean value, the intercept is really a centercept and estimates the quantile function of the boundary intensity based on the conditional mean vessel intensity.

The discrepancy correction process is shown in Fig.7. From the zoomed figure in Fig.7(b), the discrepant points, which are colored in red, are almost 
situated on the strong signal edges such as small blood vessels, bifurcates or other tissues and organs. Correction process refers to precedent and successive points' distances, which are used to estimate the discrepant mean value, and then a fine-tuning is performed within 3 pixels by quantile regression estimation. It's worth noting that this limit of band is necessary in order to avoid the extreme case under the complex texture. In the fine-tuning region, quantile regression is employed to match the most suitable pixel. The green and yellow points are the centerline points and the corrected points, respectively.

The visual comparison between the ground truth and proposed method is shown in Fig.8. As the result shows, our segmentation is very close to the ground truth in almost all parts of coronary arteries. Only in the regions of small blood vessels, the boundaries cannot be figured out, because there is no real boundaries and is impossible to fine-tune. Another deviance is in the bifurcation regions. The vascular intersection points are actually indistinct, therefore their signal intensities are not significant, and some of them are very difficult to be detected. In addition, some end arteries are lost and the segmentation of the coronary artery seems not complete. This is because the centerline is trained by the expert's delineation as the ground truth. Some centerlines in the end of artery cannot be obtained through this training process, hence our segmentation cannot extend to this region. However, the extent of the centerline can be retrained according to the actual situation of clinical demand, and it will not affect the segmentation process.

Table 2: Performance comparison of vessel segmentation

\begin{tabular}{|l|l|l|l|l|}
\hline Method & $s i(\%)$ & $f n v f(\%)$ & $f p v f(\%)$ & $t p v f(\%)$ \\
\hline Frangi's method[8] & 84.577 & 21.537 & 7.081 & 78.463 \\
\hline Vlachos's method[22] & 85.794 & 11.188 & 18.224 & 88.812 \\
\hline $\begin{array}{l}\text { Azzopardi's } \\
\text { method[10] }\end{array}$ & 90.519 & 5.649 & 14.117 & 94.351 \\
\hline Our method & 95.135 & 3.733 & 6.113 & 96.268 \\
\hline
\end{tabular}

Table 2 demonstrates the average segmentation performance comparison in false negative volume fraction $(f n v f)$, false positive volume fraction 


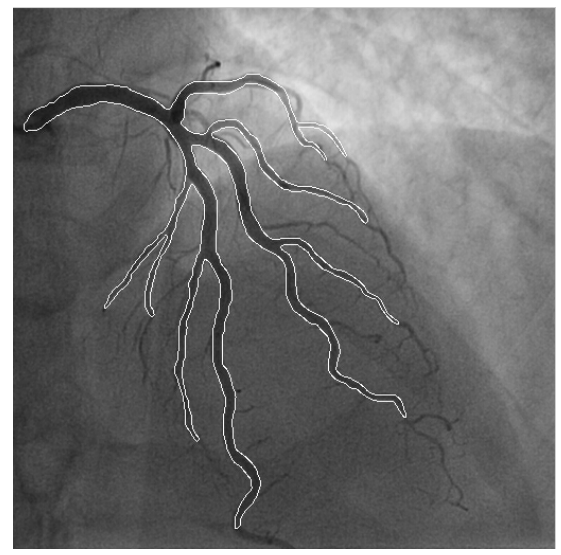

(a)

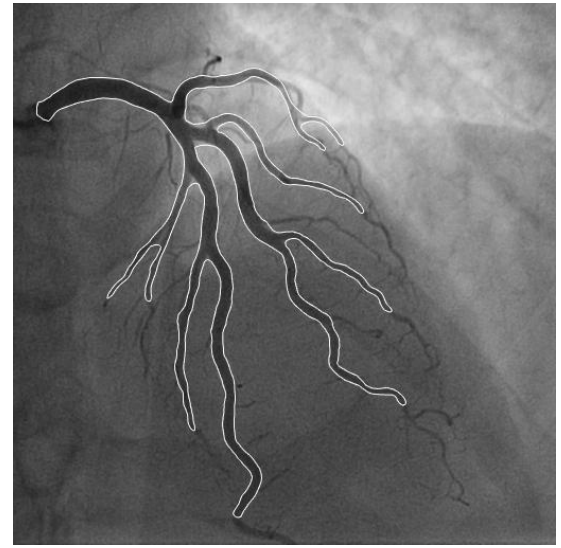

(c)

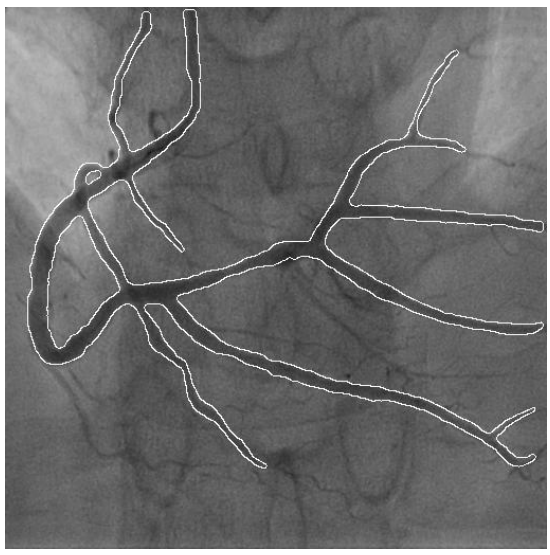

(b)

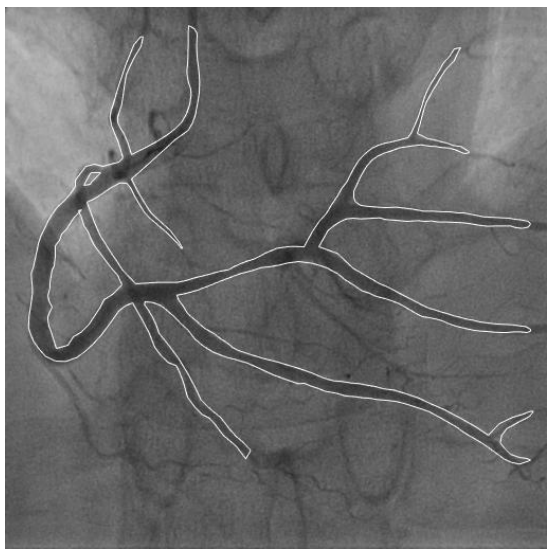

(d)

Figure 8: Segmentation result of Ground truth and proposed method: (a) Ground truth on LCA, (b) Ground truth on RCA, (c) The proposed method on LCA, (d) The proposed method on RCA 
$(f p v f)$, true positive volume fraction $(\operatorname{tpvf})$, and similarity index $(s i)$ of clinical dataset with three existing methods: Frangi's method[8], Vlachos's method[22] and Azzopardi's method[10]. These values are obtained by using the respective segmentation programs. For a fair comparison, we compare only vascular segmentation boundary and its surrounding area in various algorithms. So the same patch is employed to mask the segmentation result in all algorithms. According to the comparisons on both LCA and RCA as shown in Fig 9 and Table 2, our results are very close to the ground truth in almost all coronary arteries. The other comparative methods are relatively worse. This is mainly caused by the artifacts especially in low contrast regions and tiny vessels which should not be considered. Besides the over-segmentation, some hollow regions inside vessels may be generated by contrast agent distribution or overlapping. However, these problems can be solved well with the aid of our distance discrepancy correction and quantile regression. These hollow regions decline the performances simultaneously. In general, our method is superior to the other methods.

\section{Discussion and conclusion}

Angiogram coronary artery segmentation is a challenging issue due to its projective nature of multilayered overlapping and its discrimination difficulty between vessels and non-vascular artifacts. This paper presents a segmentation method through reliable boundary extraction by using remapping in both intensity and frequency domains. The correct boundary points can be filtered and remedied based on distance discrepancy correction and robust regression between intensity inside vessel and intensity on boundaries via respective quantiles. In addition, a patch of coarse region of interest can greatly improve the computational efficiency by employing an initial centerline, which is not necessarily very precise. The quantitative experiments also show that our method is better in comparison with existing methods.

Intensity and frequency domains are utilized in boundary extraction processing, and they have already been applied in vessel segmentation in many studies. However, they are generally used independently in the conventional methods, and the most optimal boundary may not be represented. In order to extract reliably, we rebuild a new reliable boundary map through adaptive weights on two salient maps of boundaries in both domains. The more reliable borders can enable more accurate regression. Their adaptive remapping 


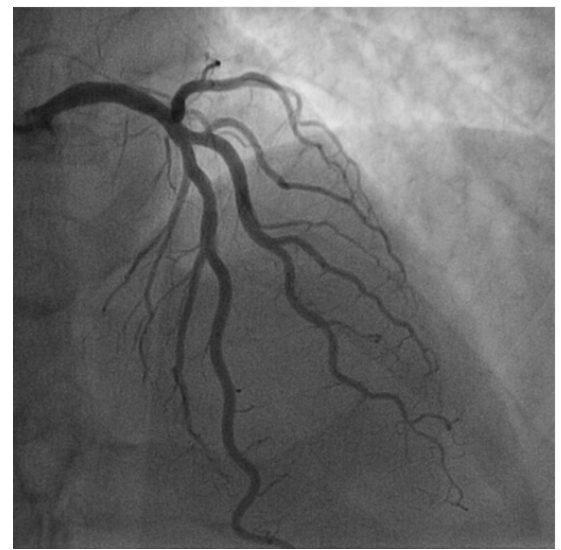

(a)

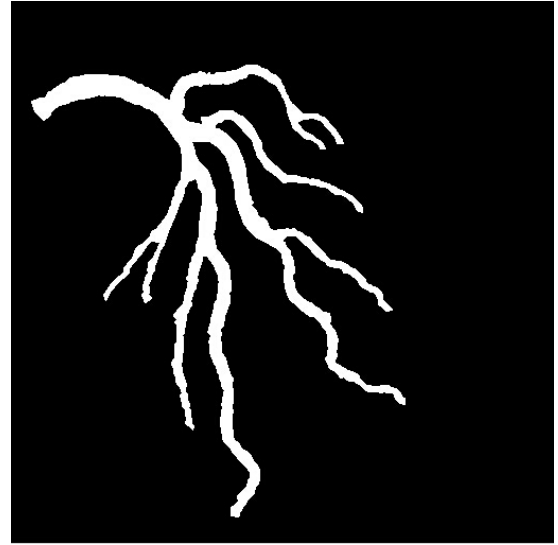

(c)

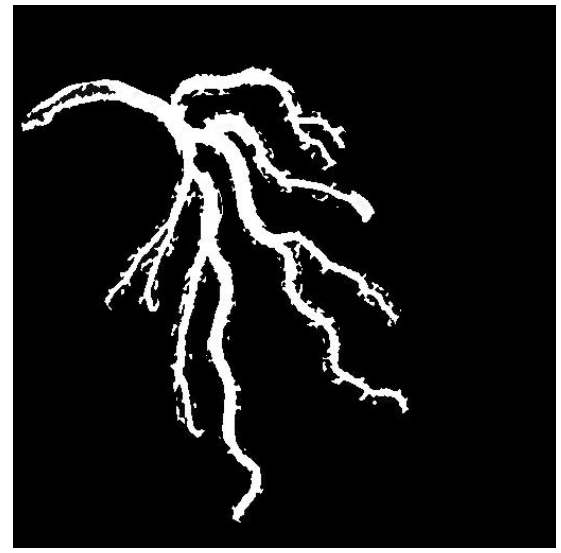

(e)

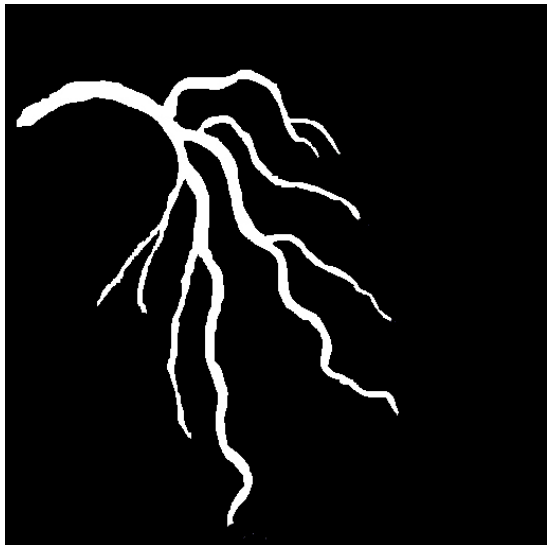

(b)

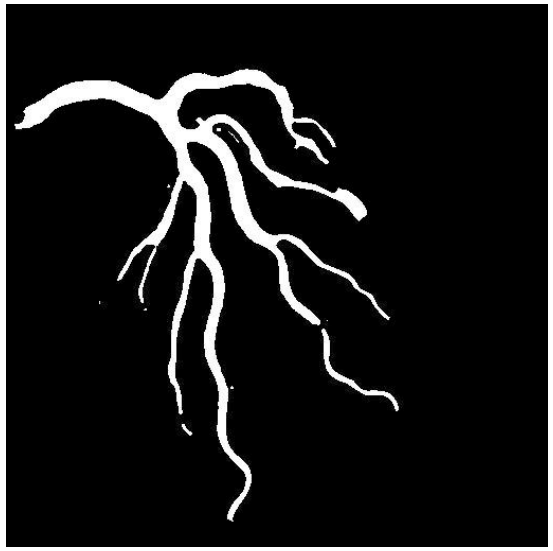

(d)

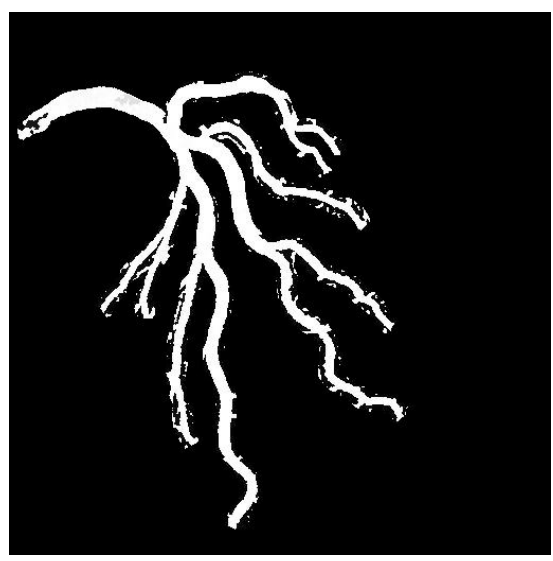

(f)

Figure 9: Comparison of binary segmentation results on LCA: (a) Original image, (b) Ground truth, (c) The proposed method, (d) Frangi's method[8], (e) Vlachos's $\operatorname{method}[22]$ and (f) Azzopardi's method[10] 


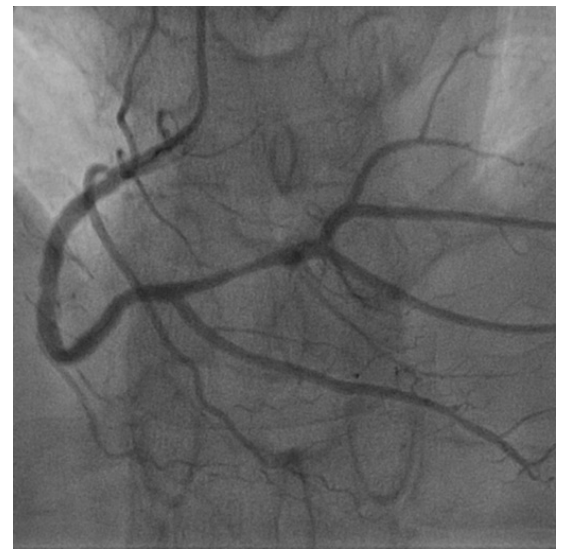

(a)

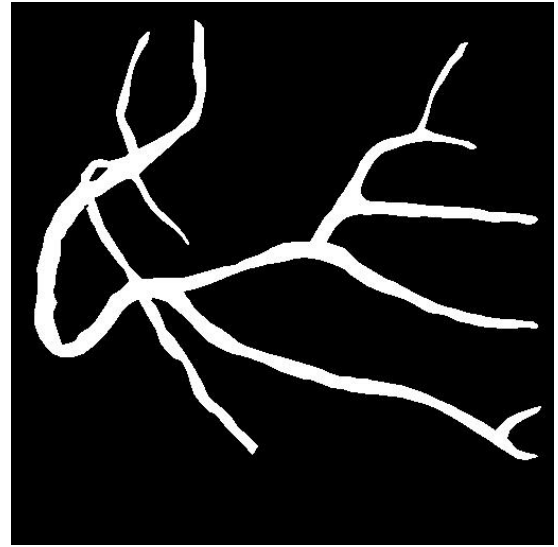

(c)

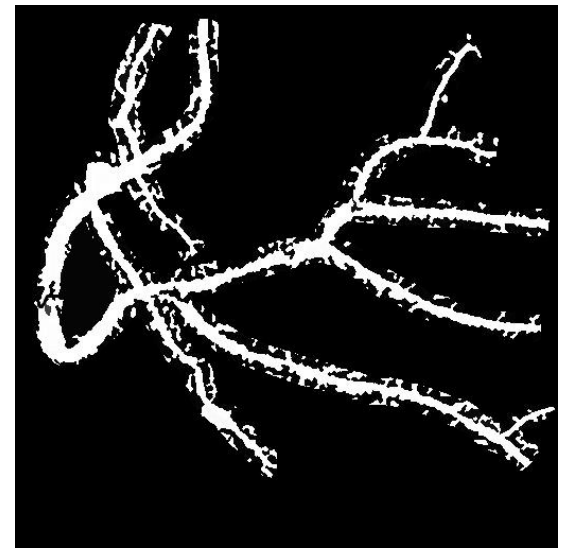

(e)

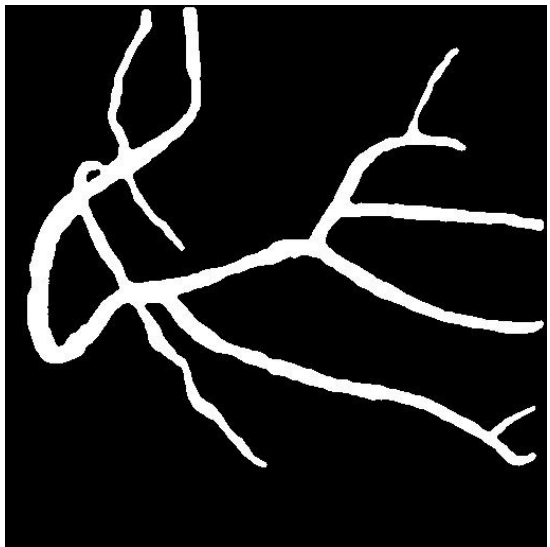

(b)

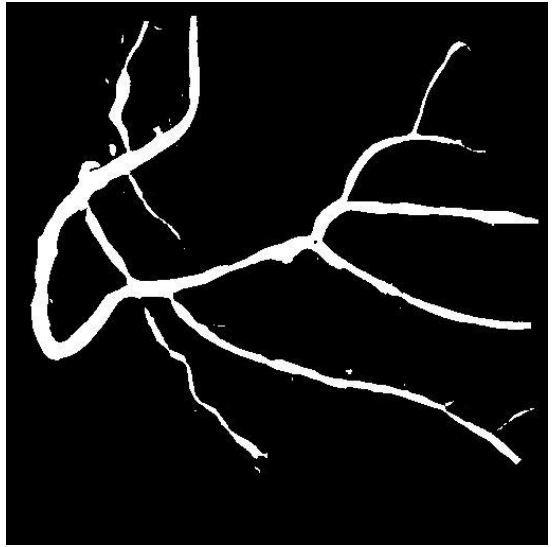

(d)

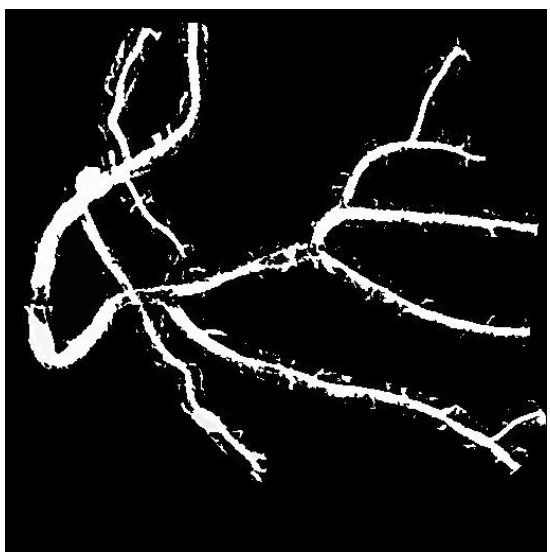

(f)

Figure 10: Comparison of binary segmentation results on RCA: (a) Original image (b) Ground truth, (c) The proposed methog, (d) Frangi's method[8], (e) Vlachos's method[22] and (f) Azzopardi's method[10] 
can eliminate dependencies on specific scenarios ,i.e, the proposed method can be easily applied to other vessels segmentation applications.

Obviously, in term of projective image, one difficulty is that it stacked with complex texture. As shown in Fig.6, we can observe that they have no entirely linear relationship between different levels of intravascular intensity and around the boundary. The optimization methods based on mean regression will result in outlier effects, which will misjudge the artifacts. Therefore the traditional linear regression model or least squares regression, which is the most basic method for coefficient estimate. They assume the random error term to be from zero mean and the same variance of the distribution. In these cases, it has excellent unbiased properties, efficiency ,etc. Unfortunately, in the real practices, this assumption is usually not able to meet. When there are serious heteroscedasticity, fat-tailed or peaks, the least squares estimate will no longer have the aforementioned good features. The quantile regression used in this work can well overcome this drawback by using the multi quantile statistics, which can make a bunch of different regression lines under different intravascular strengths. Therefore this method is more relaxed comparing with the least-squares regression, and can represent more abundant information.

In addition, a fully automatic segmentation process is also very necessary in actual clinical practice, because the vascular cases are very complex and divers, and their images are very complicated. In conventional supervised methods, the training set requires a variety of vascular shape types. Obviously, the more specifical training set is, the more effective model is trained, but the higher the demands on sample acquisition and annotations are needed. Conversely, generic or generalized training set not only is useless but also may introduce a lot of uncertainty. The proposed method not only can solve the problem of manual annotation, but also can reduce subjectivity.

Although our method shows high accuracy and robustness, there are still some limitations to be solved in the future work. In rare situations, incorrect segmentation may be occurred due to a false patch delineation cause by extreme distorting centerline. This problem can be solved by using better centerline extraction method or a centerline correction post-processing. Another drawback is in term of inexact segmentation on the missing boundary, this is because the proposed method mainly gets the border by pixel-wise processing and filtration and the missing vessel cannot be captured easily, but the ground truth is marked by observer's perception in fact. We intend to handle such problem by some useful information: the trend analysis on 
shapes or structures of vessels.

In conclusion, we have presented a robust and automatic vessel segmentation method, which combined the strength and frequency boundary information to rebuild a new boundary map with adaptive weights, and employed quantile regression to filter the artifacts robustly. The experiments demonstrate the robustness and effectiveness have been improved for vessel segmentation.

\section{Conflict of interest statement}

We confirm that 1) the submitted manuscript has been read and approved by all the listed authors; 2) all the listed authors have participated actively in the study and satisfied the criteria for authorship; 3) the protection of intellectual property associated with this study has been given due considerations; and 4) none of the authors have any conflicts of interest with regards to this research.

\section{Acknowledgment}

This work is supported, in part, by the Grant numbered 61100097 from the National Science Foundation of China.

\section{References}

[1] Roger VL, Go AS, Lloyd-Jones DM, Benjamin EJ, Berry JD, Borden WB, et al. Heart disease and stroke statistics2012 update a report from the american heart association. Circulation 2012;125(1):e2-.

[2] Swedlow JR, Goldberg I, Brauner E, Sorger PK. Informatics and quantitative analysis in biological imaging. Science 2003;300(5616):100-2.

[3] Laine AF. In the spotlight: Biomedical imaging. Biomedical Engineering, IEEE Reviews in 2008;1:4-7.

[4] Kvilekval K, Fedorov D, Obara B, Singh A, Manjunath B. Bisque: a platform for bioimage analysis and management. Bioinformatics 2010;26(4):544-52.

[5] Zhao F, Xie X. An overview of interactive medical image segmentation. Annals of the BMVA 2013;2013(7):1-22. 
[6] Lorenz C, Carlsen IC, Buzug TM, Fassnacht C, Weese J. Multi-scale line segmentation with automatic estimation of width, contrast and tangential direction in 2d and 3d medical images. In: CVRMed-MRCAS'97. Springer; 1997, p. 233-42.

[7] Sato Y, Nakajima S, Shiraga N, Atsumi H, Yoshida S, Koller T, et al. Three-dimensional multi-scale line filter for segmentation and visualization of curvilinear structures in medical images. Medical image analysis 1998;2(2):143-68.

[8] Frangi AF, Niessen WJ, Vincken KL, Viergever MA. Multiscale vessel enhancement filtering. In: Medical Image Computing and ComputerAssisted InterventationMICCAI98. Springer; 1998, p. 130-7.

[9] Kerkeni A, Benabdallah A, Manzanera A, Bedoui MH. A coronary artery segmentation method based on multiscale analysis and region growing. Computerized Medical Imaging and Graphics 2016;48:49-61.

[10] Azzopardi G, Strisciuglio N, Vento M, Petkov N. Trainable cosfire filters for vessel delineation with application to retinal images. Medical image analysis 2015;19(1):46-57.

[11] Florin C, Paragios N, Williams J. Globally optimal active contours, sequential monte carlo and on-line learning for vessel segmentation. In: Computer Vision-ECCV 2006. Springer; 2006, p. 476-89.

[12] Huang Q, Yang F, Liu L, Li X. Automatic segmentation of breast lesions for interaction in ultrasonic computer-aided diagnosis. Information Sciences 2015;314:293-310.

[13] Kolmogorov V, Zabin R. What energy functions can be minimized via graph cuts? Pattern Analysis and Machine Intelligence, IEEE Transactions on 2004;26(2):147-59.

[14] Price BL, Morse B, Cohen S. Geodesic graph cut for interactive image segmentation. In: Computer Vision and Pattern Recognition (CVPR), 2010 IEEE Conference on. IEEE; 2010, p. 3161-8.

[15] Chang H, Chen Z, Huang Q, Shi J, Li X. Graph-based learning for segmentation of 3d ultrasound images. Neurocomputing 2015;151:63244 . 
[16] Hernández-Vela A, Gatta C, Escalera S, Igual L, Martin-Yuste V, Sabaté M, et al. Accurate coronary centerline extraction, caliber estimation, and catheter detection in angiographies. Information Technology in Biomedicine, IEEE Transactions on 2012;16(6):1332-40.

[17] Cootes TF, Edwards GJ, Taylor CJ. Active appearance models. IEEE Transactions on Pattern Analysis \& Machine Intelligence 2001;(6):6815 .

[18] Van Ginneken B, Frangi AF, Staal JJ, Romeny BM, Viergever M, et al. Active shape model segmentation with optimal features. medical Imaging, IEEE Transactions on 2002;21(8):924-33.

[19] Zhang H, Wahle A, Johnson RK, Scholz TD, Sonka M. 4-d cardiac $\mathrm{mr}$ image analysis: left and right ventricular morphology and function. Medical Imaging, IEEE Transactions on 2010;29(2):350-64.

[20] Zambal S, Hladuvka J, Kanitsar A, Bühler K. Shape and appearance models for automatic coronary artery tracking. The Insight Journal $2008 ; 4$.

[21] Koulgi P, Sargin ME, Rose K, Manjunath B. Graphical model-based tracking of curvilinear structures in bio-image sequences. In: Pattern Recognition (ICPR), 2010 20th International Conference on. IEEE; 2010, p. 2596-9.

[22] Vlachos M, Dermatas E. Multi-scale retinal vessel segmentation using line tracking. Computerized Medical Imaging and Graphics 2010;34(3):213-27.

[23] Cruz-Aceves I, Oloumi F, Rangayyan RM, Aviña-Cervantes JG, Hernandez-Aguirre A. Automatic segmentation of coronary arteries using gabor filters and thresholding based on multiobjective optimization. Biomedical Signal Processing and Control 2016;25:76-85.

[24] Jolly MP, Guetter C, Guehring J. Cardiac segmentation in mr cine data using inverse consistent deformable registration. In: Biomedical Imaging: From Nano to Macro, 2010 IEEE International Symposium on. IEEE; 2010, p. 484-7. 
[25] Obara B, Fricker M, Gavaghan D, Grau V. Contrast-independent curvilinear structure detection in biomedical images. Image Processing, IEEE Transactions on 2012;21(5):2572-81.

[26] Baddeley A. Errors in binary images and an lp version of the hausdorff metric. Nieuw Archief voor Wiskunde 1992;10(4):157-83.

[27] Koenker R. Quantile regression. 38; Cambridge university press; 2005.

[28] Li Z, Zhang Y, Liu G, Shao H, Li W, Tang X. A robust coronary artery identification and centerline extraction method in angiographies. Biomedical Signal Processing and Control 2015;16:1-8.

[29] Kovesi P. Image features from phase congruency. Videre: Journal of computer vision research 1999;1(3):1-26.

[30] Felsberg M, Sommer G. The monogenic scale-space: A unifying approach to phase-based image processing in scale-space. Journal of Mathematical Imaging and vision 2004;21(1-2):5-26.

[31] Suzuki K, Horiba I, Sugie N. Linear-time connected-component labeling based on sequential local operations. Computer Vision and Image Understanding 2003;89(1):1-23. 